\title{
Generalization of Beta functions in terms of Mittag-Leffler function
}

\section{Karima Oraby, Ahmed Rizq, Mostafa Ahmed, Mohamed Khaled, Ehab Ahmed and Moamen Magdy}

Department of Mathematics and Computer Science, Faculty of Science, Suez University, Suez, Egypt.

\section{ARTICLE INFO}

Article history:

Received 20 August 2020

Received in revised form 29 August 2020

Accepted 30 August 2020

Available online 30 August 2020

\begin{abstract}
A new generalization of extended beta functions by using generalized Mittag-Leffler functions is proposed. Important properties of the generalized beta function and the integral representations are investigated. The generalization of the hypergeometric and Confluent hypergeometric functions is also introduced. Some more properties of these functions such as integral representations, differentiation formulas, transformation and summation formulas, Mellin transformations are also established.
\end{abstract}

\section{Introduction}

The Beta function play a major role in several applications of a wide variety of physical and mathematical problems. The integral representation of the classical Beta function is

$$
\begin{aligned}
\beta(a, b) & =\int_{0}^{1} t^{a-1}(1-t)^{b-1} d t \\
& =\frac{\Gamma(a) \Gamma(b)}{\Gamma(a+b)}
\end{aligned}
$$

Where the real part of $a, \mathfrak{R}(a)>0$ and $\mathfrak{R}(b)>0$.

The Gauss hypergeometric and confluent hypergeometric functions are defined by [2]

$$
F(a, b ; c ; z)=\sum_{n=0}^{\infty} \frac{(a)_{n}(b)_{n}}{(c)_{n}} \cdot \frac{z^{n}}{n !}, \quad|z|<1,
$$

and

$$
\phi(b ; c ; z)=\sum_{n=0}^{\infty} \frac{(b)_{n}}{(c)_{n}} \cdot \frac{z^{n}}{n !}, \quad|z|<1,
$$

respectively, where $(a)_{n}$ denotes the Pochhammer symbol defined by

$(a)_{n}=\frac{\Gamma(a+n)}{\Gamma(a)}=\left\{\begin{array}{rr}a(a+1) \cdots(a+n-1), & n \geq 1, \\ 1, & n=0, a \neq 0 .\end{array}\right.$

* Corresponding authors at: Suez University

E-mail addresses: koraby83@yahoo.com (Karima Oraby)
By substituting the above relation into equations (1.3), (1.4) and using the generalized binomial theorem

$$
\sum_{n=0}^{\infty}(a)_{n} \cdot \frac{(z t)^{n}}{n !}=(1-z t)^{-a}
$$

the hypergeometric and confluent hypergeometric functions can be written as integral forms, see [2]

$$
\begin{gathered}
F(a, b ; c ; z)=\frac{\Gamma(c)}{\Gamma(b) \Gamma(c-b)} \int_{0}^{1} t^{b-1}(1-t)^{c-b-1}(1 \\
-z t)^{-a} d t
\end{gathered}
$$

and

$$
\phi(b ; c ; z)=\frac{\Gamma(c)}{\Gamma(b) \Gamma(c-b)} \int_{0}^{1} t^{b-1}(1-t)^{c-b-1} e^{z t} d t
$$

respectively, where $\mathfrak{R}(c)>\Re(b)>0$ and $|\arg (1-z)|<$ $\pi$.

Recently researchers introduced many extensions of various special functions due to its applications in different fields. The recent development and properties of such extension can be found in various articles (see e.g., [1,37,9-15]). For examples, the extended beta function due to Chaudhry et al. [5] is defined by

$$
\beta(a, b ; p)=\beta_{p}(a, b)=\int_{0}^{1} t^{a-1}(1-t)^{b-1} e^{\frac{-p}{t(1-t)}} d t,
$$

where $\mathfrak{R}(a)>0, \mathfrak{R}(b)>0$ and $\mathfrak{R}(p) \geq 0$. 
The extended hypergeometric and confluent hypergeometric functions by using the definition of $\beta_{p}(a, b)$ are defined by

$$
F_{p}(a, b ; c ; z)=\sum_{n=0}^{\infty}(a)_{n} \frac{\beta_{p}(b+n, c-b)}{\beta(b, c-b)} \cdot \frac{z^{n}}{n !},
$$

and

$$
\phi_{p}(b ; c ; z)=\sum_{n=0}^{\infty} \frac{\beta_{p}(b+n, c-b)}{\beta(b, c-b)} \cdot \frac{z^{n}}{n !},
$$

respectively, where $\mathfrak{R}(a)>0, \mathfrak{R}(c)>\mathfrak{R}(b)>0, \mathfrak{R}(p) \geq$ 0 and $|z|<1$.

Also, the authors in [5] proved the following integral representations of the functions $F_{p}(a, b ; c ; z)$ and $\phi_{p}(b ; c ; z)$.

$$
F_{p}(a, b ; c ; z)=\frac{1}{\beta(b, c-b)} \int_{0}^{1} t^{b-1}(1-t)^{c-b-1}(1-z t)^{-a} e^{\frac{-p}{t(1-t)}} d t
$$

and

$$
\phi_{p}(b ; c ; z)=\frac{1}{\beta(b, c-b)} \int_{0}^{1} t^{b-1}(1-t)^{c-b-1} e^{\left(z t+\frac{-p}{t(1-t)}\right)} d t
$$

respectively, where $\mathfrak{R}(a)>0, \mathfrak{R}(c)>\mathfrak{R}(b)>0, \mathfrak{R}(p) \geq$ 0 and $|z|<1$.

Shadab et al. [15] presented a new extension of beta function by using Mittag-Leffler function as follows:

$$
\beta_{p}^{\lambda}(a, b)=\int_{0}^{1} t^{a-1}(1-t)^{b-1} E_{\lambda}\left(\frac{-p}{t(1-t)}\right) d t,
$$

where $\mathfrak{R}(a)>0, \mathfrak{R}(b)>0, \mathfrak{R}(p) \geq 0$ and $E_{\lambda}$ denots Mittage-Leffler function, which is defined by [8]

$$
E_{\lambda}(z)=\sum_{n=0}^{\infty} \frac{z^{n}}{\Gamma(\lambda n+1)}, \lambda \in \mathbb{C}, \mathfrak{R}(\lambda)>0 .
$$

Also, they defined the extended hypergeometric function and its integral representation as follows

$$
F_{p}^{\lambda}(a, b ; c ; z)=\sum_{n=0}^{\infty}(a)_{n} \frac{\beta_{p}^{\lambda}(b+n, c-b)}{\beta(b, c-b)} \cdot \frac{z^{n}}{n !},
$$

$$
=\frac{1}{\beta(b, c-b)} \int_{0}^{1} t^{b-1}(1-t)^{c-b-1}(1-z t)^{-a} E_{\lambda}\left(\frac{-p}{t(1-t)}\right) d t
$$

where $\mathfrak{R}(a)>0, \mathfrak{R}(c)>\mathfrak{R}(b)>0, \mathfrak{R}(p) \geq 0, \mathfrak{R}(\lambda)>0$ and $|z|<1$.

The extended confluent hypergeometric function and its integral representation are defined by

$$
\begin{aligned}
& \phi_{p}^{\lambda}(b ; c ; z)=\sum_{n=0}^{\infty} \frac{\beta_{p}^{\lambda}(b+n, c-b)}{\beta(b, c-b)} \cdot \frac{z^{n}}{n !}, \\
& =\frac{1}{\beta(b, c-b)} \int_{0}^{1} t^{b-1}(1-t)^{c-b-1} e^{z t} E_{\lambda}\left(\frac{-p}{t(1-t)}\right) d t
\end{aligned}
$$

where $\mathfrak{R}(c)>\mathfrak{R}(b)>0, \mathfrak{R}(p) \geq 0, \mathfrak{R}(\lambda)>0$ and $|z|<1$.

In 2018 Rahman et al. [14] extended the results of Shadab [15]. They defined the extended beta function by

$$
\beta_{p}^{\lambda, m}(a, b)=\int_{0}^{1} t^{a-1}(1-t)^{b-1} E_{\lambda}\left(\frac{-p}{t^{m}(1-t)^{m}}\right) d t
$$

where $\mathfrak{R}(a)>0, \mathfrak{R}(b)>0, \mathfrak{R}(p) \geq 0, \mathfrak{R}(\lambda)>$ $0, \mathfrak{R}(m)>0$ and $|z|<1$.

Rahman et al. [14] used $\beta_{p}^{\lambda, m}(a, b)$ to extend hypergeometric function and confluent hypergeometric function respectively, as follows

$F_{p}^{\lambda, m}(a, b ; c ; z)=\sum_{n=0}^{\infty}(a)_{n} \frac{\beta_{p}^{\lambda, m}(b+n, c-b)}{\beta(b, c-b)} \cdot \frac{z^{n}}{n !}$,

and

$\phi_{p}^{\lambda, m}(b ; c ; z)=\sum_{n=0}^{\infty} \frac{\beta_{p}^{\lambda, m}(b+n, c-b)}{\beta(b, c-b)} \cdot \frac{z^{n}}{n !}$,

where $\mathfrak{R}(a)>0, \mathfrak{R}(c)>\mathfrak{R}(b)>0, \mathfrak{R}(p) \geq 0, \mathfrak{R}(\lambda)>$ $0, \mathfrak{R}(m)>0$ and $|z|<1$.

They proved that the functions $F_{p}^{\lambda ; m}(a, b, c ; z)$ and $\phi_{p}^{\lambda, m}(b ; c ; z)$ have the following integral representations

$$
\begin{aligned}
F_{p}^{\lambda, m}(a, b ; c ; z)= & \frac{1}{\beta(b, c-b)} \int_{0}^{1}\left[t^{b-1}(1-t)^{c-b-1}\right. \\
& \left.\times(1-z t)^{-a} E_{\lambda}\left(\frac{-p}{t^{m}(1-t)^{m}}\right)\right] d t \quad(1.21)
\end{aligned}
$$


and

$$
\phi_{p}^{\lambda, m}(b ; c ; z)=\frac{1}{\beta(b, c-b)} \int_{0}^{1}\left[t^{b-1}(1-t)^{c-b-1} e^{z t} E_{\lambda}\left(\frac{-p}{t^{m}(1-t)^{m}}\right)\right] d t,
$$

where $\mathfrak{R}(c)>\mathfrak{R}(b)>0, \mathfrak{R}(p) \geq 0, \mathfrak{R}(\lambda)>0, \mathfrak{R}(m)>0$ and $|\arg (1-z)|<\pi$.

Our aim in this work is to present a new generalization of Beta function in terms of Generalized Mittag-Leffler function. We investigate its properties and its integral representations. Also, we present the generalization of hypergeometric and Confluent hypergeometric functions. Some properties of these functions such as integral representations, differentiation formulas, Mellin transformations, transformation and summation formulas are also studied.

\section{New Generalization of beta functions by using the generalized Mittag-Leffler function}

The generalized Mittag-Leffler function is used to define a new extension of Beta function, which is defined by $[8,11]$

$$
E_{\lambda, \alpha}(z)=\sum_{n=0}^{\infty} \frac{z^{n}}{\Gamma(\lambda n+\alpha)},
$$

where $\lambda, \alpha \in \mathbb{C}, \mathfrak{R}(\lambda)>0, \mathfrak{R}(\alpha)>0$. ,

We start our investigation by introducing the generalized beta function

$$
\beta_{p, \alpha}^{\lambda, m}(a, b)=\int_{0}^{1} t^{a-1}(1-t)^{b-1} E_{\lambda, \alpha}\left(\frac{-p}{t^{m}(1-t)^{m}}\right) d t,
$$

where $\mathfrak{R}(a)>0, \mathfrak{R}(b)>0, \mathfrak{R}(p) \geq 0, \mathfrak{R}(\lambda)>$ $0, \mathfrak{R}(m)>0, \mathfrak{R}(\alpha)>0$ and $E_{\lambda, \alpha}$ is the generalized Mittag-Leffler function.

The following properties can be extracted directly from the proposed extension:

1. If $\alpha=1$, then (2.2) reduces to (1.18).

2. If $m=\alpha=1$, then (2.2) reduces to (1.12).

3. If $m=\lambda=\alpha=1$, then (2.2) reduces to (1.7).

4. If $p=0$ and $m=\lambda=\alpha=1$, then (2.2) reduces to (1.1).
Theorem 2.1. The function $\beta_{p, \alpha}^{\lambda ; m}(a, b)$ has the Mellin transform

$$
M\left\{\beta_{p, \alpha}^{\lambda ; m}(a, b)\right\}=\frac{\pi}{\sin \pi s \Gamma(\alpha-s \lambda)} \beta(a+m s, b+m s)(2.3)
$$

where $\mathfrak{R}(m)>0, \mathfrak{R}(\alpha)>0, \mathfrak{R}(\lambda)>0, \mathfrak{R}(\alpha-s \lambda)>$ $0, \mathfrak{R}(a+m s)>0, \mathfrak{R}(p) \geq 0, \mathfrak{R}(b+m s)>0$.

Proof. By applying the Mellin transform [8] on both sides of (2.2), we get

$$
\begin{aligned}
& M\left\{\beta_{p, \alpha}^{\lambda ; m}(a, b)\right\}=\int_{0}^{\infty} p^{s-1} \beta_{p, \alpha}^{\lambda ; m}(a, b) d p \\
& =\int_{0}^{\infty} p^{s-1}\left(\int_{0}^{1} t^{a-1}(1-t)^{b-1} E_{\lambda, \alpha}\left(\frac{-p}{t^{m}(1-t)^{m}}\right) d t\right) d p .
\end{aligned}
$$

Interchanging the order of integrations and then setting $u=\frac{p}{t^{m}(1-t)^{m}}$, we have

$$
\begin{aligned}
M\left\{\beta_{p, \alpha}^{\lambda ; m}(a, b)\right\}= & \int_{0}^{1}\left[t^{a+m s-1}(1-t)^{b+m s-1} \times\right. \\
& \left.\int_{0}^{\infty} u^{s-1} E_{\lambda, \alpha}(-u) d u\right] d t
\end{aligned}
$$

By using the following formula [8]

$$
\int_{0}^{\infty} u^{s-1} E_{\lambda, \alpha}^{\gamma}(-\omega u) d u=\frac{\Gamma(s) \Gamma(\gamma-s)}{\Gamma(\gamma) \omega^{s} \Gamma(\alpha-s \lambda)},
$$

we obtain

$$
M\left\{\beta_{p, \alpha}^{\lambda ; m}(a, b)\right\}=\int_{0}^{1} t^{a+m s-1}(1-t)^{b+m s-1} \frac{\Gamma(s) \Gamma(1-s)}{\Gamma(\alpha-s \lambda)} d t .
$$

From the Euler's reflection formula on Gamma function

$$
\Gamma(s) \Gamma(1-s)=\frac{\pi}{\sin \pi s}, s>0,
$$

we get

$$
M\left\{\beta_{p, \alpha}^{\lambda ; m}(a, b)\right\}=\frac{\pi}{\sin \pi s \Gamma(\alpha-s \lambda)} \beta(a+m s, b+m s)
$$


Theorem 2.2. The function $\beta_{p, \alpha}^{\lambda ; m}(a, b)$ has the following integral representation

$$
\begin{aligned}
& \beta_{p, \alpha}^{\lambda, m}(a, b) \\
& =\frac{1}{2 i} \int_{\gamma-i \infty}^{\gamma+i \infty} \frac{\Gamma(a+m s) \Gamma(b+m s)}{\sin \pi s \Gamma(\alpha-s \lambda) \Gamma(a+b+2 m s)} p^{-s} d s,
\end{aligned}
$$

where $\mathfrak{R}(m)>0, \mathfrak{R}(\alpha)>0, \mathfrak{R}(\lambda)>0, \mathfrak{R}(\alpha-s \lambda)>$ $0, \mathfrak{R}(a+m s)>0, \mathfrak{R}(p) \geq 0, \mathfrak{R}(b+m s)>0$ and $\gamma>0$.

Proof. By applying the inverse Mellin transform on both sides of (2.3), we get the desired result.

Theorem 2.3. The function $\beta_{p, \alpha}^{\lambda ; m}(a, b)$ has the following integral representations

$$
\begin{array}{r}
\beta_{p, \alpha}^{\lambda, m}(a, b)=2 \int_{0}^{\pi / 2}(\cos \theta)^{2 a-1}(\sin \theta)^{2 b-1} E_{\lambda, \alpha}\left(\frac{-p}{\cos ^{m} \theta \sin ^{m} \theta}\right) d \theta, \\
\beta_{p, \alpha}^{\lambda, m}(a, b)=\int_{0}^{\infty} \frac{u^{a-1}}{(1+u)^{a+b}} E_{\lambda, \alpha}\left(\frac{-p(1+u)^{2 m}}{u^{m}}\right) d u, \quad(2.9) \\
\beta_{p, \alpha}^{\lambda, m}(a, b)=2^{1-a-b} \int_{-1}^{1}(1+u)^{a-1}(1-u)^{b-1} E_{\lambda, \alpha}\left(\frac{-4^{m} p}{\left(1-u^{2}\right)^{m}}\right) d u,
\end{array}
$$

and

$$
\begin{array}{r}
\beta_{p, \alpha}^{\lambda, m}(a, b)=(c-a)^{1-a-b} \int_{a}^{c}\left[(u-a)^{a-1}(c-u)^{b-1} \times\right. \\
\left.E_{\lambda, \alpha}\left(\frac{-p(c-a)^{2 m}}{(u-a)^{m}(c-u)^{m}}\right)\right] d u, \quad \text { (2.11) }
\end{array}
$$

where $\mathfrak{R}(m)>0, \mathfrak{R}(\alpha)>0, \mathfrak{R}(\lambda)>0, \mathfrak{R}(a)>$ $0, \Re(p) \geq 0$ and $\Re(b)>0$.

Proof. The equations (2.9) - (2.12) can be easily obtained by taking the transformation $t=\cos ^{2} \theta, t=$ $\frac{u}{1+u}, t=\frac{1+u}{2}, t=\frac{u-a}{c-a}$ in (2.2), respectively.

Theorem 2.4. The function $\beta_{p, \alpha}^{\lambda ; m}(a, b)$ satisfies the following relation

$\beta_{p, \alpha}^{\lambda, m}(a+1, b)+\beta_{p, \alpha}^{\lambda, m}(a, b+1)=\beta_{p, \alpha}^{\lambda, m}(a, b)$,

where $\mathfrak{R}(m)>0, \mathfrak{R}(\alpha)>0, \mathfrak{R}(\lambda)>0, \mathfrak{R}(a)>$ $0, \mathfrak{R}(p) \geq 0$ and $\Re(b)>0$.
Proof. The proof follows directly from (2.2) and is omitted.

Theorem 2.5. The extended beta function $\beta_{p, \alpha}^{\lambda ; m}(a, b)$ satisfies the following summation relation

$$
\beta_{p, \alpha}^{\lambda ; m}(a, 1-b)=\sum_{n=0}^{\infty} \frac{(b)_{n}}{\mathrm{n} !} \beta_{p, \alpha}^{\lambda ; m}(a+n, 1),
$$

where $\mathfrak{R}(m)>0, \mathfrak{R}(\alpha)>0, \mathfrak{R}(a)>0, \mathfrak{R}(p) \geq$ $0, \mathfrak{R}(\lambda)>0$ and $\mathfrak{R}(1-b)>0$.

Proof. Using the generalized binomial theorem

$(1-t)^{-b}=\sum_{n=0}^{\infty} \frac{(b)_{n}}{n !} t^{n}, \quad|t|<1$

in (2.2), we obtain

$\beta_{p, \alpha}^{\lambda ; m}(a, 1-b)=\int_{0}^{1} \sum_{n=0}^{\infty} \frac{(b)_{n} t^{n+a-1}}{n !} E_{\lambda, \alpha}\left(\frac{-p}{t^{m}(1-t)^{m}}\right) d t$.

Interchanging the order of summation and integration in (2.14), we get

$$
\beta_{p, \alpha}^{\lambda ; m}(a, 1-b)=\sum_{n=0}^{\infty} \frac{(b)_{n}}{\mathrm{n} !} \int_{0}^{1} t^{n+a-1} E_{\lambda, \alpha}\left(\frac{-p}{t^{m}(1-t)^{m}}\right) d t .
$$

Using (2.2) in (2.15), we get the desired result.

Theorem 2.6. The extension of beta function satisfies the following infinite summation formulas.

$$
\beta_{p, \alpha}^{\lambda ; m}(a, b)=\sum_{n=0}^{\infty} \beta_{p, \alpha}^{\lambda ; m}(a+n, b+1),
$$

where $\mathfrak{R}(m)>0, \mathfrak{R}(\alpha)>0, \mathfrak{R}(\lambda)>0, \mathfrak{R}(a)>$ $0, \mathfrak{R}(p) \geq 0$ and $\mathfrak{R}(b)>0$.

Proof. By using the binomial series expansion

$(1-t)^{b-1}=(1-t)^{b} \sum_{n=0}^{\infty} t^{n},|t|<1$

in the extended beta function (2.2), we get the desired result. 


\section{New Generalization of Extended hypergeometric and confluent hypergeometric functions}

We use the generalized extended beta function (2.2) to introduce a new generalization of an extension of extended hypergeometric and confluent hypergeometric functions.

Definition 3.1. The generalization of extended hypergeometric function is defined by

$$
F_{p, \alpha}^{\lambda, m}(a, b ; c ; z)=\sum_{n=0}^{\infty}(a)_{n} \frac{\beta_{p, \alpha}^{\lambda, m}(b+n, c-b)}{\beta(b, c-b)} \cdot \frac{z^{n}}{n !},
$$

where $\mathfrak{R}(m)>0, \mathfrak{R}(\alpha)>0, \mathfrak{R}(\lambda)>0, \mathfrak{R}(a)>$ $0, \mathfrak{R}(c)>\mathfrak{R}(b)>0, \mathfrak{R}(p) \geq 0$, and $|z|<1$.

Definition 3.2. The generalization of extended confluent hypergeometric function is defined by

$$
\phi_{p, \alpha}^{\lambda, m}(b ; c ; z)=\sum_{n=0}^{\infty} \frac{\beta_{p, \alpha}^{\lambda, m}(b+n, c-b)}{\beta(b, c-b)} \cdot \frac{z^{n}}{n !},
$$

where $\mathfrak{R}(m)>0, \mathfrak{R}(\alpha)>0, \mathfrak{R}(\lambda)>0, \mathfrak{R}(c)>\mathfrak{R}(b)>$ $0, \mathfrak{R}(p) \geq 0$, and $|z|<1$.

Remark 3.3. It is clear that

- if $\alpha=1$, then (3.1) and (3.2) reduces to relations (1.19) and (1.20) respectively.

- if $m=\alpha=1$, then (3.1) and (3.2) reduces to relations (1.13) and (1.15) respectively.

- if $\lambda=m=\alpha=1$, then (3.1) and (3.2) reduces to relations (1.8) and (1.9) respectively.

- if $\lambda=m=\alpha=1$ and $p=0$, then (3.1) and (3.2) reduces to relations (1.3) and (1.4) respectively.

Theorem 3.4. The extended hypergeometric has the following integral representation.

$$
\begin{aligned}
F_{p, \alpha}^{\lambda, m}(a, b ; c ; z)= & \frac{1}{\beta(b, c-b)} \int_{0}^{1}\left[t^{b-1}(1-t)^{c-b-1}\right. \\
& \left.\times(1-z t)^{-a} E_{\lambda, \alpha}\left(\frac{-p}{t^{m}(1-t)^{m}}\right)\right] d t,
\end{aligned}
$$

where $\mathfrak{R}(m)>0, \mathfrak{R}(\alpha)>0, \mathfrak{R}(\lambda)>0, \mathfrak{R}(a)>$ $0, \mathfrak{R}(c)>\mathfrak{R}(b)>0, \mathfrak{R}(p) \geq 0$, and $|z|<1$.

Proof. By using (2.2) in (3.1), we have

$$
\begin{gathered}
F_{p, \alpha}^{\lambda, m}(a, b ; c ; z)=\frac{1}{\beta(b, c-b)} \int_{0}^{1}\left[t^{b-1}(1-t)^{c-b-1} \times\right. \\
\left.\sum_{n=0}^{\infty}(a)_{n} \frac{(z t)^{n}}{n !} E_{\lambda, \alpha}\left(\frac{-p}{t^{m}(1-t)^{m}}\right)\right] d t
\end{gathered}
$$

By substituting

$$
\sum_{n=0}^{\infty}(a)_{n} \frac{(z t)^{n}}{n !}=(1-z t)^{-a}
$$

in (3.4), we get the desired result.

Theorem 3.5. The function $F_{p, \alpha}^{\lambda ; m}(a, b, c ; z)$ has the following integral representations

$$
\begin{gathered}
F_{p, \alpha}^{\lambda ; m}(a, b, c ; z)=\frac{1}{\beta(b, c-b)} \int_{0}^{\infty}\left[u^{b-1}(1+u)^{a-c} \times\right. \\
\left.(1+u(1-z))^{-a} E_{\lambda, \alpha}\left(\frac{-p(1+u)^{2 m}}{u^{m}}\right)\right] d u,
\end{gathered}
$$

$$
\begin{aligned}
& F_{p, \alpha}^{\lambda ; m}(a, b, c ; z) \\
& =\frac{2}{\beta(b, c-b)} \int_{0}^{\pi / 2}\left[\frac{(\sin \theta)^{2 b-1}(\cos \theta)^{2 c-2 b-1}}{\left(1-z \sin ^{2} \theta\right)^{a}} \times\right. \\
& \left.E_{\lambda, \alpha}\left(-p \sec ^{2 m} \theta \csc ^{2 m} \theta\right)\right] d \theta,
\end{aligned}
$$

and

$$
\begin{aligned}
& F_{p, \alpha}^{\lambda ; m}(a, b, c ; z) \\
& =\frac{2}{\beta(b, c-b)} \int_{0}^{\infty}\left[\frac{(\sinh \theta)^{2 b-1}(\cosh \theta)^{2 a-2 c-1}}{\left(\cosh ^{2} \theta-z \sinh ^{2} \theta\right)^{a}} \times\right. \\
& \left.E_{\lambda, \alpha}\left(-p \cosh ^{2 m} \theta \operatorname{coth}^{2 m} \theta\right)\right] d \theta,
\end{aligned}
$$

Proof. The relations (3.5) - (3.7) can be obtained by taking the transformation $t=\frac{u}{1+u}, t=\sin ^{2} \theta$ and $t=\tanh ^{2} \theta$ in (3.3) respectively. 
In the following result, we give the integral representations of extended confluent hypergeometric function.

Theorem 3.6. The extended confluent hypergeometric function has the following integral representations

$$
\begin{aligned}
\phi_{p, \alpha}^{\lambda, m}(b ; c ; z)= & \frac{1}{\beta(b, c-b)} \int_{0}^{1}\left[t^{b-1}(1-t)^{c-b-1}\right. \\
& \left.\times e^{z t} E_{\lambda, \alpha}\left(\frac{-p}{t^{m}(1-t)^{m}}\right)\right] d t,
\end{aligned}
$$

and

$$
\begin{aligned}
\phi_{p, \alpha}^{\lambda, m}(b ; c ; z)= & \frac{e^{z}}{\beta(b, c-b)} \int_{0}^{1}\left[(1-t)^{b-1} t^{c-b-1}\right. \\
& \left.\times e^{-z t} E_{\lambda, \alpha}\left(\frac{-p}{t^{m}(1-t)^{m}}\right)\right] d t,
\end{aligned}
$$

where $\mathfrak{R}(m)>0, \mathfrak{R}(\alpha)>0, \mathfrak{R}(\lambda)>0,, \mathfrak{R}(c)>$ $\mathfrak{R}(b)>0, \mathfrak{R}(p) \geq 0$, and $|z|<1$.

Proof. By using the relation (2.2) in (3.2) and then using $\sum_{n=0}^{\infty} \frac{(z t)^{n}}{n !}=e^{z}$, we get relation (3.8). To prove the relation (3.9), replacing $t$ by $(1-t)$ in relation (3.8), then we get the required result.

Theorem 3.7. The $n$-th derivative of extended hypergeometric function is given by

$$
\frac{d^{n}}{d z^{n}}\left\{F_{p, \alpha}^{\lambda, m}(a, b ; c ; z)\right\}=\frac{(a)_{n}(b)_{n}}{(c)_{n}} F_{p, \alpha}^{\lambda, m}(a+n, b+n ; c+n ; z)
$$

where $\mathfrak{R}(m)>0, \mathfrak{R}(\alpha)>0, \mathfrak{R}(\lambda)>0, \mathfrak{R}(a)>$

$0, \mathfrak{R}(b)>0, \mathfrak{R}(c)>0$ and $\mathfrak{R}(p) \geq 0$.

Proof. Differentiating (3.1) with respect to $z$, we have

$$
\frac{d}{d z}\left\{F_{p, \alpha}^{\lambda, m}(a, b ; c ; z)\right\}=\sum_{n=1}^{\infty} n(a)_{n} \frac{\beta_{p, \alpha}^{\lambda, m}(b+n, c-b)}{\beta(b, c-b)} \cdot \frac{z^{n-1}}{n !} .
$$

Replacing $n$ by $n+1$ (3.11), we get .

$\frac{d}{d z}\left\{F_{p, \alpha}^{\lambda, m}(a, b ; c ; z)\right\}=\sum_{n=0}^{\infty}(a)_{n+1} \frac{\beta_{p, \alpha}^{\lambda, m}(b+n+1, c-b)}{\beta(b, c-b)} \cdot \frac{z^{n}}{n !}$

by using the relation

$B(b, c-b)=\frac{c}{b} B(b+1, c-b),(\Re(c)>\Re(b)>0)$

and

$$
(a)_{n+1}=a(a+1)_{n}
$$

Yields

$\frac{d}{d z}\left\{F_{p, \alpha}^{\lambda, m}(a, b ; c ; z)\right\}=\frac{a b}{c} F_{p, \alpha}^{\lambda, m}(a+1, b+1 ; c+1 ; z)$

Again, differentiating with respect to $z$, we have

$$
\begin{aligned}
& \frac{d^{2}}{d z^{2}}\left\{F_{p, \alpha}^{\lambda, m}(a, b ; c ; z)\right\}= \\
& \quad=\frac{a(a+1) b(b+1)}{c(c+1)} F_{p, \alpha}^{\lambda, m}(a+2, b+2 ; c+2 ; z)
\end{aligned}
$$

Continuing up to $n$-times, we get the required result.

Theorem 3.8. The $n$-th derivative of confluent extended hypergeometric function is given by

$$
\frac{d^{n}}{d z^{n}}\left\{\phi_{p, \alpha}^{\lambda, m}(b ; c ; z)\right\}=\frac{(b)_{n}}{(c)_{n}} \phi_{p, \alpha}^{\lambda, m}(b+n ; c+n ; z),
$$

where $\mathfrak{R}(m)>0, \mathfrak{R}(\alpha)>0, \mathfrak{R}(\lambda)>0,, \mathfrak{R}(c)>$ $\mathfrak{R}(b)>0, \mathfrak{R}(p) \geq 0$, and $|z|<1$.

Proof. Applying the similar way used in Theorem 3.7, we get the required result.

Theorem 3.9. The Mellin transform of the generalized hypergeometric function is given by

$$
\begin{aligned}
M\left\{F_{p, \alpha}^{\lambda, m}(a, b ; c ; z)\right\} & =\frac{\pi \beta(b+m s, c+m s-b)}{\sin (\pi s) \Gamma(\alpha-s \lambda) \beta(b, c-b)} \times \\
& F(a, b+m s ; c+2 m s ; z) .
\end{aligned}
$$

where $\mathfrak{R}(b+m s)>0, \mathfrak{R}(\alpha)>0, \mathfrak{R}(\lambda)>0, \mathfrak{R}(\alpha-$ $s \lambda)>0, \mathfrak{R}(a)>0, \mathfrak{R}(c+m s-b)>0$, and $\mathfrak{R}(p) \geq 0$.

Proof. By applying Mellin transform on both sides of (3.3), we obtain

$$
\begin{gathered}
M\left\{F_{p, \alpha}^{\lambda, m}(a, b ; c ; z)\right\}=\frac{1}{\beta(b, c-b)} \int_{0}^{\infty} p^{s-1}\left[\int_{0}^{1} t^{b-1}(1-t)^{c-b-1}\right. \\
\left.\times(1-z t)^{-a} E_{\lambda, \alpha}\left(\frac{-p}{t^{m}(1-t)^{m}}\right) d t\right] d p .
\end{gathered}
$$

Interchanging the order of integrations in above equation, we have 


$$
\begin{aligned}
& M\left\{F_{p, \alpha}^{\lambda, m}(a, b ; c ; z)\right\}=\frac{1}{\beta(b, c-b)}\left(\int_{0}^{1} t^{b-1}(1-t)^{c-b-1}\right. \\
&\left.\times(1-z t)^{-a} \int_{0}^{\infty} p^{s-1} E_{\lambda, \alpha}\left(\frac{-p}{t^{m}(1-t)^{m}}\right) d p\right) d t
\end{aligned}
$$

Let. $u=\frac{p}{t^{m}(1-t)^{m}}$. Then we have

$$
\begin{aligned}
M\left\{F_{p, \alpha}^{\lambda, m}(a, b ; c ; z)\right\} & \frac{1}{\beta(b, c-b)}\left(\int_{0}^{1} t^{b+m s-1}(1-t)^{c+m s-b-1}\right. \\
& \left.\times(1-z t)^{-a} \int_{0}^{\infty} u^{s-1} E_{\lambda, \alpha}(-u) d u\right) d t
\end{aligned}
$$

By using (2.4) and (2.6) in above equation, we get the desired result.

Theorem 3.10. The function $F_{p, \alpha}^{\lambda ; m}(a, b, c ; z)$ has the following integral representation

$$
\begin{aligned}
& F_{p, \alpha}^{\lambda ; m}(a, b, c ; z) \\
& =\frac{1}{2 i \beta(b, c-b)} \int_{\gamma-i \infty}^{\gamma+i \infty}\left[\frac{\Gamma(b+m s) \Gamma(c+m s-b)}{\sin \pi s \Gamma(\alpha-s \lambda) \Gamma(b+2 m s)}\right. \\
& \left.\times \quad F(a, b+m s ; c+2 m s ; z) p^{-s}\right] d s
\end{aligned}
$$

where $\mathfrak{R}(b+m s)>0, \mathfrak{R}(\alpha)>0, \mathfrak{R}(\lambda)>0, \mathfrak{R}(\alpha-$ $s \lambda)>0, \mathfrak{R}(a)>0, \mathfrak{R}(c+m s-b)>0$, and $\mathfrak{R}(p) \geq 0$.

Proof. By taking the inverse Mellin transform on both sides of (3.16), we get the required result.

In similar way, we can prove the following theorems for extended confluent hypergeometric functions.

Theorem 3.11. The extended confluent hypergeometric function has the following Mellin transform

$$
\begin{aligned}
M\left\{\phi_{p, \alpha}^{\lambda, m}(b ; c ; z)\right\}= & \frac{\pi \beta(b+m s, c+m s-b)}{\sin (\pi s) \Gamma(\alpha-s \lambda) \beta(b, c-b)} \times \\
& \phi(b+m s ; c+2 m s ; z) .
\end{aligned}
$$

where $\mathfrak{R}(b+m s)>0, \mathfrak{R}(\alpha)>0, \mathfrak{R}(\lambda)>0, \mathfrak{R}(\alpha-$ $s \lambda)>0, \mathfrak{R}(c+m s-b)>0$, and $\mathfrak{R}(p) \geq 0$.

Theorem 3.12. The function $\phi_{p, \alpha}^{\lambda ; m}(b, c ; z)$ has the following integral representation

$$
\begin{aligned}
& \phi_{p, \alpha}^{\lambda ; m}(b, c ; z) \\
& =\frac{1}{2 i \beta(b, c-b)} \int_{\gamma-i \infty}^{\gamma+i \infty}\left[\frac{\Gamma(b+m s) \Gamma(c+m s-b)}{\sin \pi s \Gamma(\alpha-s \lambda) \Gamma(c+2 m s)}\right. \\
& \left.\times \phi(b+m s ; c+2 m s ; z) p^{-s}\right] d s
\end{aligned}
$$

where $\mathfrak{R}(b+m s)>0, \mathfrak{R}(\alpha)>0, \mathfrak{R}(\lambda)>0, \mathfrak{R}(\alpha-$ $s \lambda)>0, \Re(c+m s-b)>0$, and $\Re(p) \geq 0$.

The transformation and summation formulas for the generalized extended hypergeometric and confluent hypergeometric functions are obtained in the following results.

Theorem 3.13. The following transformation for extended hypergeometric function holds true

$$
F_{p, \alpha}^{\lambda ; m}(a, b, c ; z)=(1-z)^{-a} F_{p, \alpha}^{\lambda ; m}\left(a, c-b, b ; \frac{z}{z-1}\right)
$$

where $\mathfrak{R}(a)>0, \mathfrak{R}(\alpha)>0, \mathfrak{R}(\lambda)>0, \mathfrak{R}(c)>\mathfrak{R}(b)>$ $0, \mathfrak{R}(m)>0, \mathfrak{R}(p) \geq 0$ and $\arg |1-z|<\pi$.

Proof. Replacing $t$ by $(1-t)$ in (3.3), we get the desired result.

Theorem 3.14. The following transformation for extended confluent hypergeometric function holds true

$$
\phi_{p, \alpha}^{\lambda, m}(b ; c ; z)=e^{z} \phi_{p, \alpha}^{\lambda, m}(c-b ; c ; z)
$$

where $\mathfrak{R}(\alpha)>0, \mathfrak{R}(\lambda)>0, \mathfrak{R}(c)>\mathfrak{R}(b)>0, \mathfrak{R}(m)>$ $0, \mathfrak{R}(p) \geq 0$ and $\arg |1-z|<\pi$.

Proof. The proof follows from (3.8) and (3.9).

Theorem 3.15. The relation between the extended hypergeometric function and extended beta function is given by

$$
F_{p, \alpha}^{\lambda ; m}(a, b, c ; z)=\frac{\beta_{p, \alpha}^{\lambda ; m}(b, c-b-a)}{\beta(b, c-b)},
$$

where $\mathfrak{R}(a)>0, \mathfrak{R}(\alpha)>0, \mathfrak{R}(\lambda)>0, \mathfrak{R}(c)>\mathfrak{R}(b)>$ $0, \mathfrak{R}(m)>0, \mathfrak{R}(p) \geq 0$ and $\arg |1-z|<\pi$.

Proof. Taking $z=1$ in relation (3.3) we get the desert result.

\section{Conclusion}

In this paper, we introduce a new generalization of Beta functions in terms of Generalized Mittag-Leffler function. We investigate its properties and its integral representations. Also, we present the generalization of hypergeometric and Confluent hypergeometric functions. Some properties of these functions such as integral representations, differentiation formulas, Mellin transformations, transformation and summation formulas are also studied. Our results are extended and generalized the results of $[5-7,9,10,14,15]$. 


\section{References}

[1] 1 P. Agarwal, J. Choi and S. Jain, Extended hypergeometric functions of two and three varaibles, Commun. Korean Math. Soc. 30 (2015), pp. 403-414.

[2] G. E. Andrews, R. Askey and R. Roy, Special Functions, Encyclopedia of Mathematics and its Applications, vol. 71, Cambridge University Press, Cambridge, 1999.

[3] M. Arshad, J. Choi, S. Mubeen, K. S. Nisar and G. Rahman, A new extension of the Mittag Leffler function, Commun. Korean Math. Soc. 33 (2018), pp. 549-560.

[4] M. A. Chaudhry and S. M. Zubair, Generalized incomplete gamma functions with applications, J. Comput. Appl. Math. 55 (1994), pp. 99-124.

[5] M. A. Chaudhry, A. Qadir, M. Rafique and S. M. Zubair, Extension of Eulers beta function, J. Comput. Appl. Math. 78 (1997), pp 19-32.

[6] J. Choi, A. K. Rathie and R. K. Parmar, Extension of extended beta, hypergeometric and confluent hypergeometric functions, Honam Math. J., 36 (2014), pp. 357-385.

[7] D.M. Lee, A.K. Rathie, R.K. Parmar and Y.S. Kim, Generalization of extended beta function, hypergeometric and confluent hypergeometric functions, Honam Math. J., 33 (2011), pp. 187- 206.

[8] A. M. Mathai and H. J. Haubold, Spacial functions for applied Scientists, Springer, (2008).

[9] S. Mubeen, G. Rahman, K. S. Nisar, J. Choi and M. Arshad, An extended beta function and its properties, Far East J. Math. Sci., 102(2017), pp. 1545-1557.

[10] E. Ozergin, M. A. Ozarslan and Abdullah Altin, Extension of gamma, beta and hypergeometric functions, J. Comput. Appl. Math., 235 (2011), pp. 4601-4610.

[11] M. A. Ozarslan and B. Yilmaz,The extended Mittag-Leffler function and its properties, Journal of Inequalities and Applications, 2014:85, (2014).

[12] R. K. Parmar, Extended T-hypergeometric functions and associated properties, C. R. Acad.Sci. Paris, Ser. I353 (2015), pp. 421-426.

[13] R. K. Parmar, A new generalization of gamma, beta, hypergeometric and confluent hypergeometric functions, Matematiche (Catania), (2013), pp. 3352.

[14] G. Rahman, S. Mubeen, K. S. Nisar, A new generalization of extended beta and hypergeometric functions, Journal of Fractional Calculus and Applications, Vol 11(2) July (2020), pp. 32-44.

[15] M. Shadab, S. Jabee and J. Choi, An extension of beta function and its application, Far East J. Math. Sci., 103(2018), pp. 235-251. 